\title{
Constraints on the exosphere of CoRoT-7b ${ }^{\star}$
}

\author{
E. W. Guenther ${ }^{1,2}$, J. Cabrera ${ }^{3,4}$, A. Erikson ${ }^{3}$, M. Fridlund ${ }^{5}$, H. Lammer ${ }^{6}$, A. Mura ${ }^{7}$, H. Rauer ${ }^{3,9}$, J. Schneider ${ }^{4}$, \\ M. Tulej ${ }^{8}$, Ph. von Paris ${ }^{3}$, and P. Wurz ${ }^{8}$
}

1 Thüringer Landessternwarte Tautenburg, Sternwarte 5, 07778 Tautenburg, Germany e-mail: guenther@tls-tautenburg.de

2 Instituto de Astrofísica de Canarias, C/Vía Láctea, s/n, 38205 La Laguna (Tenerife), Spain

3 Institute of Planetary Research, German Aerospace Center, Rutherfordstraße 2, 12489 Berlin, Germany

${ }^{4}$ LUTH, Observatoire de Paris, CNRS, Université Paris Diderot, 5 place Jules Janssen, 92190 Meudon, France

5 Research and Scientific Support Department, ESTEC/ESA, PO Box 299, 2200 AG Noordwijk, The Netherlands

6 Space Research Institute, Austrian Academy of Science, Schmiedlstraße. 6, 8042 Graz, Austria

7 Istituto di Fisica dello Spazio Interplanetario-CNR, Rome, Italy

8 Physics Institute, University of Bern, Bern, Switzerland

9 Zentrum für Astronomie und Astrophysik (ZAA), Technische Universität Berlin (TUB), Hardenbergstraße 36, 10623 Berlin, Germany

Received 26 April 2010 / Accepted 30 September 2010

\section{ABSTRACT}

Context. The small radius and high density of CoRoT-7b implies that this transiting planet belongs to a different species than all transiting planets previously found. Current models suggest that this is the first transiting rocky planet found outside the solar system. Given that the planet orbits a solar-like star at a distance of only $4.5 R_{*}$, it is expected that material released from its surface may then form an exosphere.

Aims. We constrain the properties of the exosphere by observing the planet in- and out-of-transit. Detecting the exosphere of CoRoT$7 \mathrm{~b}$ would for the first time allow us to study the material originating in the surface of a rocky extrasolar planet. We scan the entire optical spectrum for any lines originating from the planet, focusing particularly on spectral lines such as those detected in Mercury and Io in our solar system.

Methods. Since lines originating in the exosphere are expected to be narrow, we observed CoRoT-7b at high resolution with UVES on the VLT. By subtracting the two spectra from each other, we search for emission and absorption lines originating in the exosphere of CoRoT-7b.

Results. In the first step, we focus on Ca I, Ca II, and Na, because these lines have been detected in Mercury. Since the signal-to-noise ratio $(\mathrm{S} / \mathrm{N})$ of the spectra is as high as 300 , we derive firm upper limits for the flux-range between $1.6 \times 10^{-18}$ and $3.2 \times 10^{-18} \mathrm{~W} \mathrm{~m}^{-2}$. For $\mathrm{CaO}$, we find an upper limit of $10^{-17} \mathrm{~W} \mathrm{~m}^{-2}$. We also search for emission lines originating in the plasma torus fed by volcanic activity and derive upper limits for these lines. In the whole spectrum we finally try to identify other lines originating in the planet. Conclusions. Except for $\mathrm{CaO}$, the upper limits derived correspond to $2-6 \times 10^{-6} L_{*}$, demonstrating the capability of UVES to detect very weak lines. Our observations certainly exclude the extreme interpretations of data for CoRoT-7b, such as an exosphere that emits 2000 times as brightly as Mercury.

Key words. planetary systems - planets and satellites: atmospheres - techniques: spectroscopic planets and satellites: individual: CoRoT-7b

\section{Introduction}

Studies of transiting extra-solar planets are of key importance for understanding the nature of planets, because it is possible to derive their mass, diameter, and hence their density. Observations of transits even allow us to detect the atmosphere of the planets. Up to now, studies of the atmospheres of extrasolar planets have concentrated on giant gaseous planets. The detection of transiting planets of low mass and small radius now permits us to examine the atmosphere of planets that are possibly rocky planets.

CoRoT-7b is an extrasolar planet of a radius of $1.58 \pm$ $0.10 R_{\text {Earth }}$ orbiting a G9V star at a distance of only $4.5 R_{*}$ (original values: Léger et al. 2009; new values: Bruntt et al. 2010). The mass is $6.9 \pm 1.4 M_{\text {Earth }}$, and the density is $\rho=$ $9.6 \pm 2.7 \mathrm{~g} \mathrm{~cm}^{-3}$ (original value: Queloz et al. 2009; new value: Hatzes et al. 2010). The density is thus even higher than Earth

* Based on observations obtained at the European Southern Observatory at Paranal, Chile in program 384.C-0820(A). $\left(\rho=5.515 \mathrm{~g} \mathrm{~cm}^{-3}\right)$, Venus $\left(\rho=5.243 \mathrm{~g} \mathrm{~cm}^{-3}\right)$, or Mercury $\left(\rho=5.427 \mathrm{~g} \mathrm{~cm}^{-3}\right)$.

Although the density of this planet even exceeds those of the rocky planets of our solar system, it may not necessarily be a rocky planet. However, its low mass, small radius, and high density indicate that it certainly belongs to a different species than the gaseous planets studied so far. It would thus be exciting to determine its surface composition, and assess whether it has an atmosphere or not.

Future instruments such as SEE-COAST (now called SPICES) are designed to study the atmospheres and surface compositions of planets down to a few Earth-radii (Schneider et al. 2009). The instrumentation study SEARCH (Mohler et al. 2010) demonstrates that an 8-m-class space telescope equipped with a highly sophisticated spectropolarization device would be able to study the atmospheres and surface composition of extrasolar planets of similar size to the Earth. While the prospects for such studies are high, it will take decades until such missions are 
operate. However, there is already a fair chance of detecting material released from the surface of rocky extrasolar planets using existing instrumentation.

If a sufficient quantity of material is released from the surface of the planet into space, its surface composition may be studied by performing spectroscopy when it is both in- and outof-transit.

While the exosphere of gaseous extrasolar planets has been detected in hydrogen as well as heavier elements (Vidal-Madjar et al. 2003; Vidal-Madjar et al. 2004; Lecavelier des Etangs et al. 2010; Linsky et al. 2010; Holmström, et al. 2008; Eckenbäck et al. 2010; Fossati 2010), no attempts have yet been made to detect the exosphere of a rocky extrasolar planet.

Our observations represent the first attempt to detect the exosphere of a rocky extrasolar planet. We present UVES observations taken in- and out-of-transit that we use to search for emission lines originating in material released from the surface of the planet.

\section{What we might expect to find}

Whether or not material from the surface of the planet is released depends not only on the properties of the planet but also those of the star. The host star has an effective temperature $T_{\text {eff }}=5250 \pm 60 \mathrm{~K}$, a mass of $M_{*}=0.91 \pm 0.03 M_{\odot}$, and a radius of $R_{*}=0.82 \pm 0.04 R_{\odot}$ (Bruntt et al. 2010). Although the rotation period of the host star is at 23 days quite close to that of the Sun, the star is more active than the Sun. The total chromospheric radiative loss in the $\mathrm{Ca} I \mathrm{H}, \mathrm{K}$ lines in units of the bolometric luminosity is $\log R_{\mathrm{HK}}=-4.601 \pm 0.05$, which implies a significantly higher activity level than the Sun, for which $\log R_{\mathrm{HK}}=-4.901$ (Baliunas et al. 1996). On the basis of this activity index, the age of the star is estimated to be in the range 1.2-2.3 Gyr. Given that the star is quite similar to the Sun, apart from being slightly more active, it is reasonable to assume that its stellar wind is also quite similar to that of the Sun. Because the star is an active G-star, we can safely assume that there is a stellar wind that will interact with the planets.

Before we go ahead and try to detect the material released from the surface, we discuss how likely the planet is to be rocky. Until now, we know about the planet's mass and radius, not its composition. Assuming a rocky planet, Valencia et al. (2010) could reproduce the original values of the radius and mass of $1.68 \pm 0.10 R_{\text {Earth }}$ and $4.8 \pm 0.8 M_{\text {Earth }}$, respectively, given in Léger et al. (2009) and Queloz et al. (2009). However, to reproduce the relatively low density given in these articles they had to assume that the planet is significantly depleted in iron compared to the Earth. The authors argue that this is unlikely and that it is more likely that it has the same composition as the Earth. If this were the case, the mass and radius would be $5.6 M_{\text {Earth }}$ and $1.59 R_{\text {Earth }}$, respectively. Interestingly, the new values of mass and radius are $6.9 \pm 1.4 M_{\text {Earth }}$ and $1.58 \pm 0.10 R_{\text {Earth }}$, respectively, which are perfectly consistent with those of a rocky planet with the composition of the Earth. Using realistic values for the heating efficiencies, and also taking the evolution of the EUV flux of the host star into account, Leitzinger et al. (2010) showed that CoRoT-7b was always a rocky planet and is not the eroded core of a gas giant. Given all these results, it is justified to assume that CoRoT-7b is a rocky planet.

Given that we do not have a planet like CoRoT-7b in our solar system, the structure, surface composition, and how much material is released from its surface is not known. For this reason, we scan the whole optical spectrum for lines originating in the planet. Nevertheless, it makes sense to discuss which lines we might expect. CoRoT-7b is certainly not like either Mercury or Io but these objects do have some similarities to CoRoT-7b. We use these analogies to discuss which lines we should be looking for.

In Sect. 2 we discuss the species we expect to find. In Sect. 3, we report on the observations, in Sect. 4 we present the limits derived for $\mathrm{Na}, \mathrm{Ca}, \mathrm{Ca}^{+}, \mathrm{CaO}, \mathrm{S}^{+}, \mathrm{S}^{2+}$, and $\mathrm{O}^{2+}$, and in Sect. 4 we discuss the results obtained.

\subsection{The Mercury analogy}

To some extent, the local environment of CoRoT-7b is an extreme version of Mercury, as its distance to the host star is 23 times smaller than that of Mercury. Hence the planet receives about 250-370 times more radiation than Mercury. In addition, the stellar wind is more intensive than at Mercury, because the host star is more active than the Sun, and the planet closer to its host star. Could it also have a Mercury-like exosphere, and if so what would we expect to observe?

The gaseous envelope of Mercury was discovered by the Mariner 10 spacecraft. Since Mercury's envelope is collisionless, it resembles an exosphere where the exobase is coincident with the planet's surface. An exosphere is the collisionless, outermost layer of an atmosphere of the planet where atoms, ions, or molecules can escape into space. Mariner 10 detected UV emission from the exosphere of three atomic elements: $\mathrm{H}, \mathrm{He}$, and $\mathrm{O}$ (Broadfoot et al. 1976). Three other elements (Na, K, and Ca) were later discovered by ground-based observations (Potter \& Morgan 1985; Bida et al. 2000). The exosphere of Mercury is extremely thin. The surface pressure is only $10^{-15} \mathrm{bar}$, and the total mass $\leq 1000 \mathrm{~kg}$, which is tiny compared to the atmosphere of the Earth with its mass of $5 \times 10^{18} \mathrm{~kg}$. It is believed that the exosphere of Mercury is derived in large part from the surface materials (Wurz \& Lammer 2003; Wurz et al. 2009).

A combination of for instance impact vaporization (from in-falling material), volatile evaporation, photon-stimulated desorption, and sputtering, releases material from the surface to form the exosphere. The ground-based observations by Bida et al. (2000) reveal CaI emission in the resonance line at $4226.74 \AA$. Observations performed during the transit of Mercury in 2003 detected additionally sodium emission lines (Na I D $D_{1,2}$ ), which allowed Schleicher et al. (2004) to trace the extent of the exosphere of Mercury above the planet's limb. Potter et al. (2002) observed a sodium-tail in the anti-sunward direction extending ten's of planetary radii.

Mura et al. (2010) applied their model of Mercury to CoRoT$7 \mathrm{~b}$ and found that surface elements should in this case be released into space. By analogy with Mercury, the authors postulate that there is a sodium-tail extending ten's of planetary radii in the anti-stellar direction. According to these authors, the radiation pressure on the sodium atoms is about a factor of 100 higher than the gravitational force, which causes the sodium-tail to be almost perfectly aligned with the star-planet axis and the cross-section of the sodium-tail to be only slightly larger than the planet itself. In addition, Mura et al. (2010) postulate the existence of $\mathrm{a} \mathrm{Ca}^{+}$-ion tail. Exospheric $\mathrm{Ca}$ is ionized very rapidly by the stellar photon field and the $\mathrm{Ca}^{+}$-ions are guided by the stellar wind. Given the high transverse velocity of the planet of $218 \mathrm{~km} \mathrm{~s}^{-1}$ with respect to the stellar wind (estimated to be around $200 \mathrm{~km} \mathrm{~s}^{-1}$ ), which flows radially away from the star, the ion tail is expected to be inclined by about $45^{\circ}$ with respect to the star-planet line. Thus, during transit the $\mathrm{Ca}^{+}$tail is also inclined by $45^{\circ}$ to the line of sight. According to these authors, the Ca II lines are seen in absorption during the transit. They find that the 
Table 1. Observing log.

\begin{tabular}{lccccc}
\hline \hline Name & Date & $\begin{array}{c}\text { UT } \\
\text { mid point }\end{array}$ & $\begin{array}{c}\text { Exposure } \\
\text { time [s] }\end{array}$ & $\begin{array}{c}\text { HJD } \\
\text { mid-point }\end{array}$ & $\begin{array}{c}\text { Phase } \\
\text { mid-point }\end{array}$ \\
\hline transit Ia & 2009.12 .28 & $02: 43: 14$ & 970 & 2455193.61851 & 0.9899 \\
transit Ib & 2009.12 .28 & $03: 00: 06$ & 970 & 2455193.63023 & 0.0036 \\
transit Ic & 2009.12 .28 & $03: 15: 04$ & 970 & 2455193.64062 & 0.0158 \\
\hline off Ia & 2009.12 .28 & $06: 45: 20$ & 970 & 2455193.78664 & 0.1868 \\
off Ib & 2009.12 .28 & $07: 02: 12$ & 970 & 2455193.79835 & 0.2006 \\
off Ic & 2009.12 .28 & $07: 19: 09$ & 970 & 2455193.81012 & 0.2144 \\
\hline transit II & 2010.01 .03 & $02: 29: 17$ & 970 & 2455199.60885 & 0.0077 \\
off II & 2010.01 .07 & $02: 46.33$ & 970 & 2455203.62067 & 0.7076 \\
\hline
\end{tabular}

coma has an extension of several $R_{\odot}$. We conclude that CoRoT$7 \mathrm{~b}$ may have an exosphere and given the extreme conditions on CoRoT-7b the exosphere may also be detectable.

We note that the cometary tail of a hot Jupiter HD 209458 b has apparently already been detected (Linsky et al. 2010).

\subsection{The lo analogy}

A Mercury-like planet is not the only possibility for CoRoT-7b. Because the planet is heated not only by the radiation of the star but also the strong tidal forces, it may resemble Io. Barnes et al. (2010) pointed out that the tidal heating may be as strong as the radiative heating by the star, if the orbit of CoRoT-7b is slightly eccentric $\left(e \geq 10^{-5}\right)$. This eccentricity could be driven by CoRoT-7c. In this case, CoRoT-7b may be dominated by volcanism and rapid resurfacing, and possibly even have a plasma torus like Io. These types of planets had already been suggested before the discovery of CoRoT-7b by Briot \& Schneider (2008). However, even if CoRoT-7b had strong volcanic activity, it is not immediately obvious that it would also have a plasma torus similar to that of Io. Since the torus of Io emits lines in the wavelength regime covered by our spectra, we can also search for them. Prominent lines originating from the torus of Io are the $\mathrm{Na} I \mathrm{D}_{1,2}$-lines and the forbidden emission lines [S II] $(\lambda \lambda 6716$, $6731 \AA)$, [S III] ( $\lambda 6312 \AA$ ), and [O III] ( $\lambda 5007 \AA$ ) (e.g. Brown et al. 2008; Rauer et al. 1993; Thomas 1996).

\section{Observations}

High-resolution spectroscopy of CoRoT-7b taken in- and out-oftransit is ideal for detecting the exosphere or the plasma torus, because the lines are expected to be very narrow, like those of Mercury. The broadening of the lines is mainly due to the change in the radial velocity of the planet during the course of the observations.

Two sets of observations were carried out in service mode with UVES on ESO VLT UT-2 (KUEYEN). In the night of 2728 December 2009, CoRoT-7b was observed for 50 min during transit and for 50 min out-of-transit. The out-of-transit observations were taken about $3.5 \mathrm{~h}$ after the end of the transit, to make sure that the tail is no longer in front of the star. The second data-set was taken on 3 January 2010 and 7 January 2010. The in-transit observations were taken on 3 January 2010, and the out-of-transit observations on 7 January 2010. In each case, the observing time was only $15 \mathrm{~min}$. In the following, we indicate the first data set with an $I$, and the second data set with an $I I$.

During our observations, we used the standard 390+580 setting, which covers the wavelength region from $3270 \AA$ to $6820 \AA$. Unfortunately, orders that are only partly covered by the detector, cannot be used for this type of analysis. Thus, the blue channel effectively covers only the spectral range from 3290 to $4520 \AA$. There are two detectors for the red channel, which cover the wavelength regions from 4780 to $5740 \AA$, and from 5820 to $6760 \AA$.

We used a slit-width of 0.6 arcsec, which provides a resolution of $\lambda / \Delta \lambda=65000$. Apart from the differences in exposure time, the second data-set has the disadvantage that the in- and out-of-transit observations were taken on different nights. Given the difference in exposure times and signal-to-noise ratio $(\mathrm{S} / \mathrm{N})$, the data-set $I$ was used to search for the lines from the planet, and data-set $I I$ to confirm whether they were found. Another interesting property of data-set $I I$ is that its out-of-transit observations were taken at phase 0.7 , compared to 0.2 for the data-set $I$.

A series of IRAF routines were used to remove both the bias and scattered light, to flat-field the spectra, and then to extract and wavelength-calibrate them. The sky background was removed by extracting the spectrum of the night-sky along the slit and subtracting it from the spectrum of the star. For the wavelength calibration, we used the ThAr spectra taken after the observations and performed a global fit using 886 and $1523 \mathrm{ThAr}$ lines for the two CCDs of the red arm and 2518 ThAr lines for the blue arm. The mid-points of the observation, and their corresponding phases are given in Table 1 . The transit lasts about $1.3 \mathrm{~h}$, or 0.0635 in phase.

\section{Results}

\subsection{Sodium}

Because the NaD-lines have been detected at Mercury and in the plasma torus of Io, we select these lines for our study. Figure 1 shows the coadded spectrum of CoRoT-7b of data-set $I$. The wavelength is in the rest frame of the star. The full line is the spectrum taken in-transit, and the dashed line is the spectrum taken out-of-transit. The $\mathrm{S} / \mathrm{N}$ of the coadded spectra of the first data-set are about 150 per pixel. Since there are 4 pixel per resolution element, the resulting $\mathrm{S} / \mathrm{N}$ per resolution element is 300 for the data-set $I$, and 180 for data-set $I I$. The quality of the spectra is so high that it is straightforward to discern even a small difference between the spectra after resampling the spectra. The two NaD-lines are very normal absorption lines as expected for a G-type star. There is no evidence for any additional emission or absorption line.

In the next step, we subtracted the out-of-transit spectrum from the in-transit spectrum (middle line in Fig. 1). The variance in the difference between the two spectra is $8.5 \times$ $10^{-19} \mathrm{~W} \mathrm{~m}^{-2} \AA^{-1}$, thus about 100 times smaller than the flux of the continuum in each spectrum, which is $8.2 \times 10^{-17} \mathrm{~W} \mathrm{~m}^{-2} \AA^{-1}$. Because the planet changes its radial velocity by $55 \mathrm{~km} \mathrm{~s}^{-1}$ during the exposure, we expect any line emitted by the planet to have (at least) this width. Thus, we resampled the spectrum to 


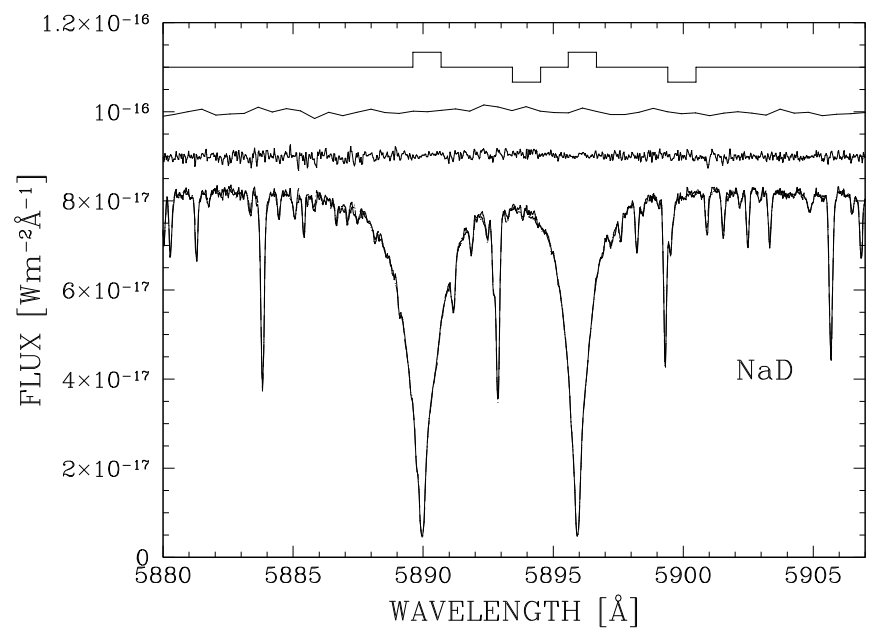

Fig. 1. Spectra taken in- (full line) and out-of-transit (dashed line) in the range of the Na D-lines. The two lines above the spectrum represent the difference between the two spectra, and the difference after resampling to the expected line-width of $55 \mathrm{~km} \mathrm{~s}^{-1}$.

this resolution, which reduced the noise yet further (Fig. 1). After resampling, the resolution is decreased to $\lambda / \Delta \lambda=5500$, and the $\mathrm{S} / \mathrm{N}$ increased by a factor of three. After subtracting the spectra from each other, we then performed two different analysis, one to search for narrow lines at full resolution $(\lambda / \Delta \lambda=65000)$, and a second to search for broad features at medium resolution $(\lambda / \Delta \lambda=5500)$.

Do we expect to see an emission or an absorption feature in Fig. 1? If we define $\alpha$ as the angle between the line of sight and a tail driven by the radiation of the star, then $\alpha$ changes from $-7^{\circ}$ to $8^{\circ}$ during transit $I$. During the out-of-transit observations for the same night, $\alpha$ changes from $65^{\circ}$ to $80^{\circ}$. For the data-set $I I, \alpha$ is $3^{\circ}$ for the in-transit observations and $105^{\circ}$ for the out-of-transit observations, respectively.

This means that we observe almost directly along the radiatively driven sodium-tail during the transit. In the course of the out-of-transit observations, we see the tail almost from the side. In both cases, the cross-section of the sodium-tail is not much wider than the planet (Mura et al. 2010). During transit, we expect to detect the absorption of stellar light, because we are aligned with the star-planet line (phase angle around $180^{\circ}$ ). Out-of-transit, we expect to detect emission from the tail of the observations (phase angle around $90^{\circ}$ ). Thus, if we subtracted the out-of-transit observations from the in-transit observations, we would also observe an absorption feature if the planet had a sodium tail best viewed from the side (out-of-transit). The line at the top of Fig. 1 indicates the expected position and width of either the absorption or emission feature originating in the planet. The box pointing downwards is the position of the sodium lines during the out-of-transit observations, and the box pointing upwards marks the expected position of the sodium lines originating in the planet during transit. A close inspection shows neither an emission nor absorption feature in Fig. 1. Thus, the NaD-lines from CoRoT-7b are not detected in the current set of data.

The $3 \sigma$ upper limits of $\mathrm{NaD}$ are given in Tables 2 and 3 . Assuming that the radiation is emitted spherically symmetrically, and using the distance of $150 \mathrm{pc}$, we derive the upper limits to the total emitted flux for the spectral lines and list them in the third column of Tables 2 and 3. Given the total luminosity of the star of $1.9 \times 10^{26} \mathrm{~W}$, we also derive the upper limits for the lines divided by the luminosity of the star. For the NaD-lines, we conclude that we might have detected a line that either absorbs
Table 2. $3 \sigma$-upper limits to the fluxes for the first data set.

\begin{tabular}{lccc}
\hline \hline Line & $\begin{array}{c}\text { Measured } \\
\mathrm{W} \mathrm{m}^{-2}\end{array}$ & $\begin{array}{c}\text { Total } \\
\mathrm{W}\end{array}$ & $\begin{array}{c}\text { Fraction } \\
\text { of } L_{*}\end{array}$ \\
\hline $\mathrm{Ca}$ II K & $2.9 \times 10^{-18}$ & $7.7 \times 10^{20}$ & $4.0 \times 10^{-6}$ \\
$\mathrm{Ca}$ II H & $3.2 \times 10^{-18}$ & $8.7 \times 10^{20}$ & $4.6 \times 10^{-6}$ \\
$\mathrm{Ca} \mathrm{I} \mathrm{4227}^{-18}$ & $3.9 \times 10^{-18}$ & $1.0 \times 10^{21}$ & $5.4 \times 10^{-6}$ \\
$\mathrm{Na} \mathrm{D}_{1}$ & $1.6 \times 10^{-18}$ & $4.2 \times 10^{20}$ & $2.2 \times 10^{-6}$ \\
$\mathrm{Na} \mathrm{D}_{2}$ & $1.6 \times 10^{-18}$ & $4.2 \times 10^{20}$ & $2.2 \times 10^{-6}$ \\
$\mathrm{CaO}$ & $1.0 \times 10^{-17}$ & $2.6 \times 10^{21}$ & $1.4 \times 10^{-5}$ \\
\hline$[\mathrm{O} \mathrm{III}] 5007$ & $4.4 \times 10^{-18}$ & $1.2 \times 10^{21}$ & $6.1 \times 10^{-6}$ \\
{$[\mathrm{~S} \mathrm{III}] 6312$} & $3.5 \times 10^{-18}$ & $9.6 \times 10^{20}$ & $5.0 \times 10^{-6}$ \\
{$[\mathrm{~S} \mathrm{II}] 6716$} & $3.1 \times 10^{-18}$ & $8.4 \times 10^{20}$ & $4.4 \times 10^{-6}$ \\
{$[\mathrm{~S} \mathrm{II}] 6731$} & $3.1 \times 10^{-18}$ & $8.4 \times 10^{20}$ & $4.4 \times 10^{-6}$ \\
\hline
\end{tabular}

Table 3. $3 \sigma$-upper limits to the fluxes for the second data set.

\begin{tabular}{lccc}
\hline \hline Line & $\begin{array}{c}\text { Measured } \\
\mathrm{W} \mathrm{m}^{-2}\end{array}$ & $\begin{array}{c}\text { Total } \\
\mathrm{W}\end{array}$ & $\begin{array}{c}\text { Fraction } \\
\text { of } L_{*}\end{array}$ \\
\hline $\mathrm{Ca}$ II K & $8.5 \times 10^{-18}$ & $2.2 \times 10^{21}$ & $1.2 \times 10^{-5}$ \\
$\mathrm{Ca}$ II H & $1.0 \times 10^{-17}$ & $2.8 \times 10^{21}$ & $1.4 \times 10^{-5}$ \\
$\mathrm{Ca} \mathrm{I}_{4} 227$ & $7.7 \times 10^{-18}$ & $2.1 \times 10^{21}$ & $1.0 \times 10^{-5}$ \\
$\mathrm{Na} \mathrm{D}_{1}$ & $3.4 \times 10^{-18}$ & $9.2 \times 10^{20}$ & $4.8 \times 10^{-6}$ \\
$\mathrm{Na} \mathrm{D}_{2}$ & $3.4 \times 10^{-18}$ & $9.2 \times 10^{20}$ & $4.8 \times 10^{-6}$ \\
$\mathrm{CaO}$ & $3.0 \times 10^{-17}$ & $7.8 \times 10^{21}$ & $4.1 \times 10^{-5}$ \\
\hline$[\mathrm{O}$ III $] 5007$ & $2.1 \times 10^{-17}$ & $5.8 \times 10^{21}$ & $3.0 \times 10^{-5}$ \\
{$[\mathrm{~S} \mathrm{III}] 6312$} & $8.8 \times 10^{-18}$ & $2.4 \times 10^{21}$ & $1.2 \times 10^{-5}$ \\
{$[\mathrm{~S} \mathrm{II}] 6716$} & $6.1 \times 10^{-18}$ & $1.6 \times 10^{21}$ & $8.5 \times 10^{-6}$ \\
{$[\mathrm{~S}$ II] 6731 } & $6.1 \times 10^{-18}$ & $1.6 \times 10^{21}$ & $8.5 \times 10^{-6}$ \\
\hline
\end{tabular}

or emits only $2.2 \times 10^{-6} L_{*}$. One may argue that if the emission originated in a torus, we would not detect it after subtracting the out-of-transit spectrum from the in-transit spectrum, because the emission would originate in both. However, the emission along the torus of Io is not constant so would not cancel out. A line emitted by a ring or a torus that has the same distance from the star as the planet would have a width of $430 \mathrm{~km} \mathrm{~s}^{-1}$. The corresponding spectral line would thus be very broad and have the shape of a spectral line like Io (Thomas 1996). Prior to the subtraction of the two spectra, we inspected the spectra carefully but did not find any broad emission line. The hypothesis of a sodium torus is thus excluded.

\subsection{Neutral Ca}

Because Bida et al. (2000) detected the Ca I emission-line at $4226.74 \AA$ we also searched for this line. Figure 2 shows the spectra taken in- and out-of-transit, and Fig. 3 their differences. The line at the top of Fig. 3 shows the expected position and width of the lines originating from the planet in the same way as shown in Fig. 1.

\subsection{The $\mathrm{Ca}^{+}$-ion}

Assuming that CoRoT-7b resembles Mercury, a considerable amount of $\mathrm{Ca}$ is expected in the exosphere, and given the very short photo ionisation time of $\mathrm{Ca}$ it should soon be ionized (Mura et al. 2010). Thus, the Ca II H, K lines are expected to be seen in the spectra. According to these authors, the $\mathrm{Ca}^{+}$-ions would be guided by the stellar wind and the ion-tail would be at an angle of about $45^{\circ}$ with respect to the star-planet line. Thus, we expect an absorption feature in the spectrum of the difference (Fig. 4). As before, we resampled the spectrum to a resolution of $55 \mathrm{~km} \mathrm{~s}^{-1}$. 


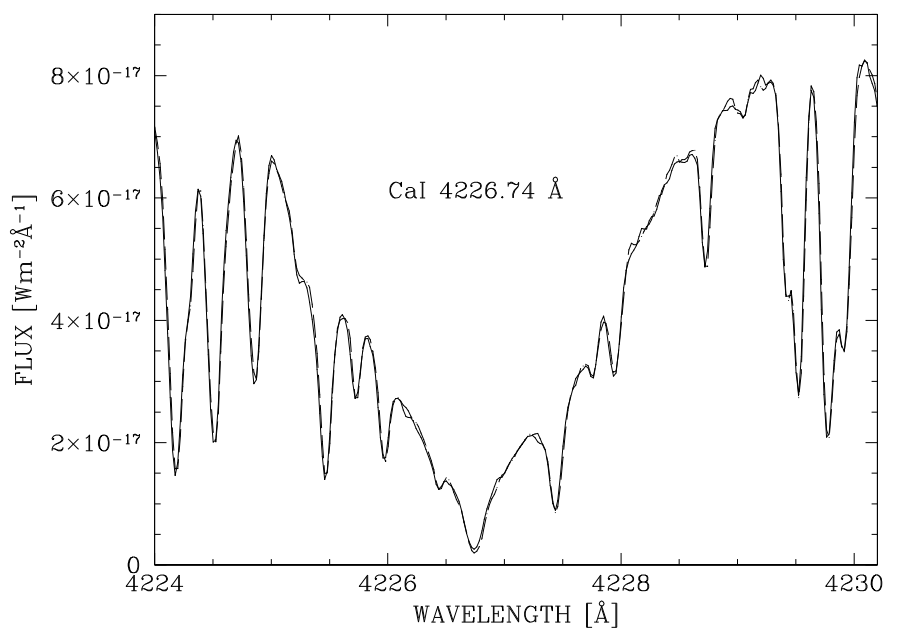

Fig. 2. Spectra taken in- (full line) and out-of-transit (dashed line) in the range of the Ca I line at $4226.74 \AA$.

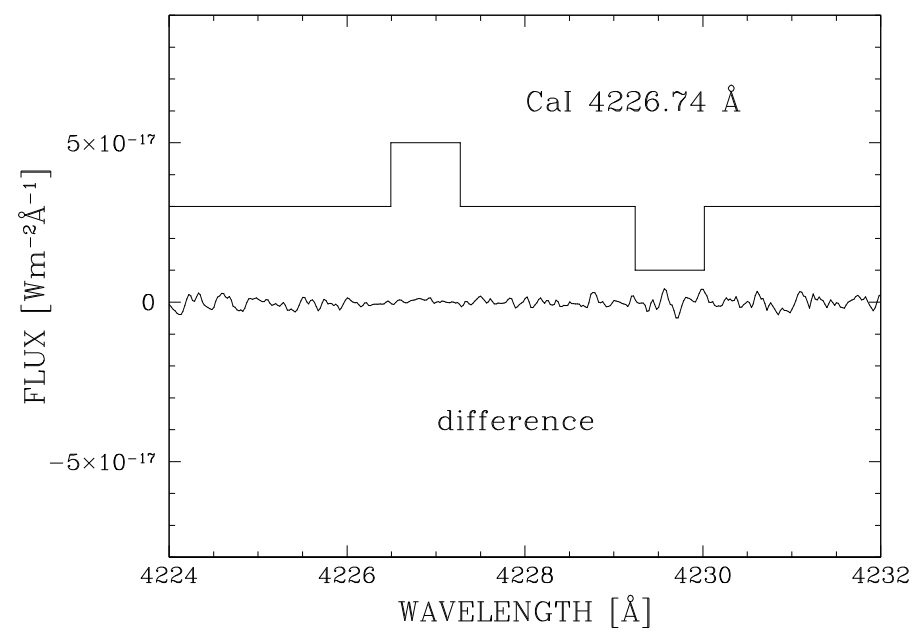

Fig. 3. Difference between the two spectra shown in Fig. 2, together with the expected position of the lines from CoRoT-7b. Box pointing upwards indicates the expected position of the line during transit. Box pointing downwards indicates the expected position of the line in the out-of-transit observation.

Figure 4 shows the spectra taken in- and out-of-transit prior to the subtraction. We again detect neither an emission nor absorption line originating in the planet. The upper limits are given in Tables 2 and 3.

\subsection{The $\mathrm{CaO}$ molecule}

The next series of plots show the analysis for $\mathrm{CaO}$ (Figs. 5 and 6). Since $\mathrm{CaO}$ is a molecule, it has a rich spectrum of transitions. Figure 6 shows the calculated $\mathrm{CaO}$ spectrum as a dashed line overplotted on the difference spectrum for the in-transit and out-of-transit observations. The theoretical $\mathrm{CaO}$ spectrum shown in Fig. 6 was calculated assuming a temperature of $2500 \mathrm{~K}$ and folding the resolution of our UVES observations. Thus, the individual lines are not resolved but there should be a very prominent peak, which is not seen. The relatively high upper limits of $1.0 \times 10^{-17}$ and $3.0 \times 10^{-17} \mathrm{~W} \mathrm{~m}^{-2}$ is caused by the feature not being very broad, and we assume that the peak of the feature has to be $3 \sigma$ above the noise level.

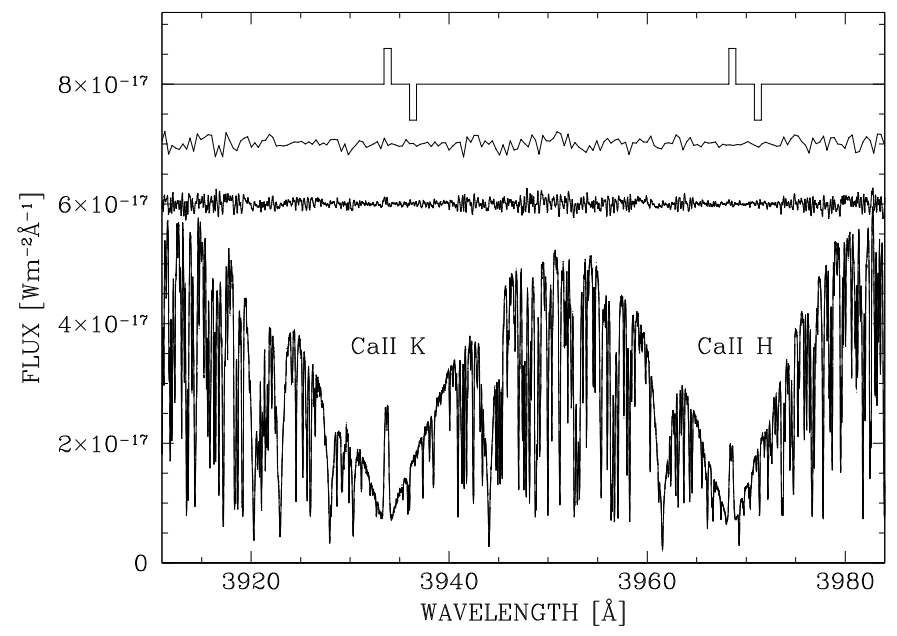

Fig. 4. Same as Fig. 1 but for the Ca II H and K lines.

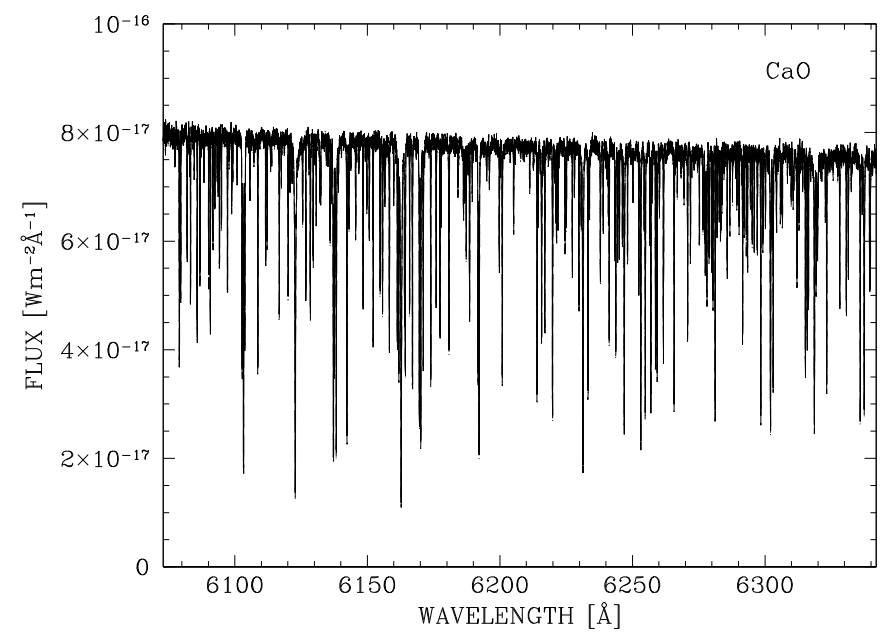

Fig. 5. Spectra taken in- (full line) and out-of-transit (dashed line) in the range of the $\mathrm{CaO}$ lines.

\subsection{The $\mathrm{S}^{+}, \mathrm{S}^{2+}$, and $\mathrm{O}^{2+}$-ions}

In addition to the hypothesis that CoRoT-7b resembles Mercury, we also tested the hypothesis that more closely resembles Io. In this case, we would expect to detect NaD-lines but as we have already seen above, there is no detectable $\mathrm{NaD}$-emission from the planet. Other possibilities are [S II] $(\lambda \lambda 6716,6731 \AA)$, [S III] ( $\lambda 6312 \AA)$, and [O III] $(\lambda 5007 \AA)$, which we also failed to detect (e.g. Brown et al. 2008; Rauer et al. 1993; Thomas 1996). The upper limits to the fluxes of these lines are given in Tables 2 and 3 .

\subsection{Upper limits for the whole spectrum}

To maximise our chances of detection, we carried out the same kind of analysis for the whole UVES spectrum. Figure 7 shows the upper limits derived for each order. Because of the blazefunction of the spectrograph, there are more photons at the centre of each order than at the edges. This means that the noise level and the upper limits are higher at the edges of an order than its centre. We always derived the upper limits for a whole order to avoid making Fig. 7 too complicated. Given the form of the blaze-function, this effect is greater in the blue than the red. However, the specific spectral line may be at the centre of 


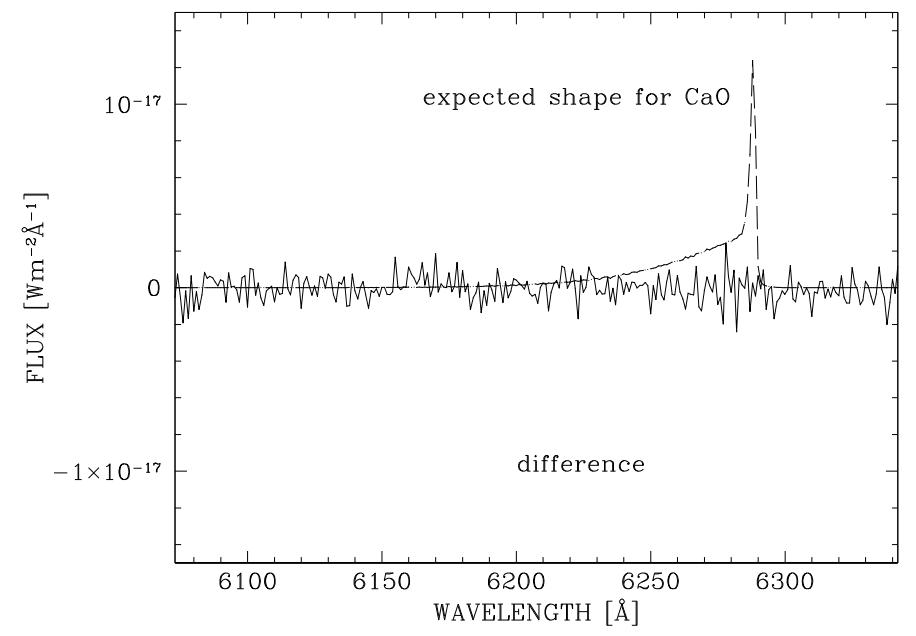

Fig. 6. Difference of the spectra taken in- and out-of-transit shown in Fig. 5 after resampling the spectrum to a resolution of $55 \mathrm{~km} \mathrm{~s}^{-1}$. The dashed line demonstrates how the $\mathrm{CaO}$-lines would look.

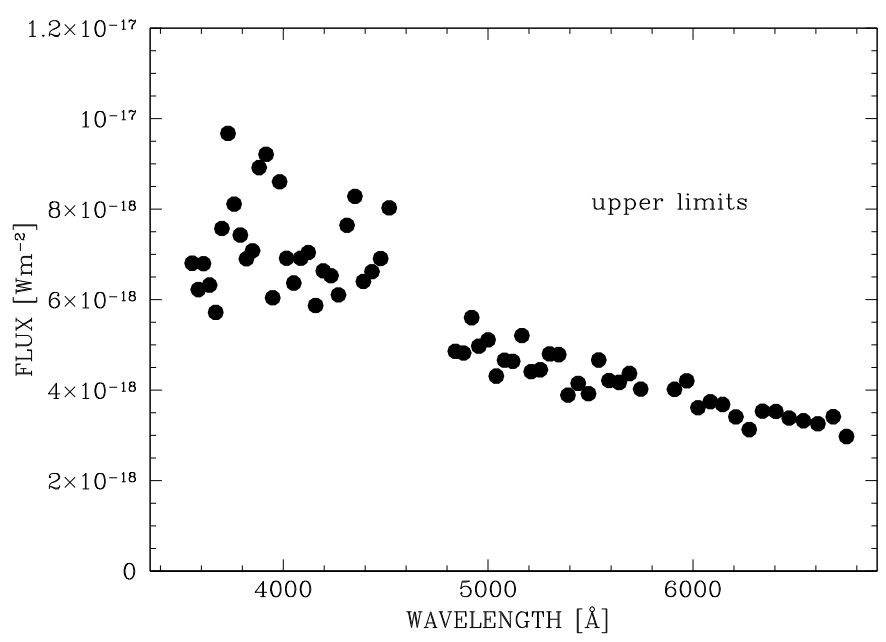

Fig. 7. 3 $\sigma$-upper limits to the fluxes of lines per spectral order.

one order and closer to the edges of another one. Since the overlap of the orders is larger in the blue than in the red, the effect of the blaze-function is partly compensated. This explains why the upper limits for $\mathrm{Ca}$ II H, K are not significantly poorer than those for $\mathrm{NaD}$. The result of the analysis is that no emission or absorption line originating in the planet is found in the whole spectrum.

\section{Discussion and conclusions}

CoRoT-7b is the first small transiting extrasolar planet with a density comparable to, or even higher than those of Mercury, Earth, Venus, and Mars. It is thus reasonable to assume that it is rocky. The radius of the planet is $1.58 \pm 0.09 R_{\text {Earth }}$, or $4.1 \pm$ $0.2 R_{\text {Mercury. }}$ Given its small distance from the star, the surface of the planet is expected to be very hot. Léger et al. (2009) estimated the temperature to be in the range $1800-2600 \mathrm{~K}$. Barnes et al. (2010) pointed out that there is tidal heating possibly as strong as the heating by the star. These properties make it reasonable to assume that material from the surface could be released into space to form an atmosphere as in the case of Mercury or Io. If this material were detected, we would be able to perform mineralogy of a rocky, extrasolar planet. We have searched for the emission and absorption lines of CoRoT-7b using spectra taken in- and out-of-transit. Although our measurements are very sensitive, we could not detect any spectral lines originating in the planet.

How well do our upper limits compare to the emission observed for Mercury? For the Ca I $4227 \AA$ A-line, Bida et al. (2000) found a column emission intensity of $384 \times 10^{6}$ photons $\mathrm{cm}^{-2} \mathrm{~s}^{-1}$, corresponding to $5.1 \times 10^{17} \mathrm{~W}$. Our upper limit is $10^{21} \mathrm{~W}$, or about 2000 times higher than the line-flux detected for Mercury. Since the surface area of CoRoT-7b is about 17 times larger than that of Mercury, and CoRoT-7b receives about 250-370 times more radiation than Mercury, our upper limit is even lower than the Caemission observed at Mercury if we scale accordingly. However, since the photoionisation of $\mathrm{Ca}$ atoms is very fast for CoRoT-7b, we expect to observe $\mathrm{Ca}^{+}$-emission, which is not the case.

Given the extreme conditions on this planet and the sensitivity of our measurements, it is surprising that we have been unable to detect the exosphere. On the other hand, since we do not have a planet like this in our solar system, we have had to make a number of assumptions. We assumed for example that one side of the planet is always facing the star. If this were not the case, the conditions would be less extreme than we think. Our knowledge of the stellar wind for this star is also very limited. Although we are certain that this star has a wind, and we are also certain that the plasma flow will dictate the flow of ions originating in the planet, we know neither the velocity nor density of the stellar wind. Thus, we do not know the volume shape of the interacting region between the stellar plasma and the ions from the planet.

We have also assumed that the surface of CoRoT-7b is similar to that of Mercury. In this case, lines from $\mathrm{Ca}$ II and $\mathrm{Na}$ would be expected. But how well is this assumption justified? At the moment, all we know is that the measured density corresponds to a planet with a composition similar to Earth. If the surface composition were very different, it would not be surprising if we did not detect these lines. This is a possibility, since Schaefer \& Fegley (2009) suggested that "super-Earths" do not have large quantities of $\mathrm{Ca}$ and $\mathrm{Na}$. In this case, the planet should form a Mg-ion-tail, which is not detectable in the optical regime. Schaefer \& Fegley (2009) considered a surface composition for super-Earth's that evolved in a different way to Mercury's. Their model calculations indicate that volatile elements such as $\mathrm{H}, \mathrm{C}$, $\mathrm{N}, \mathrm{S}$, and $\mathrm{Cl}$ have been lost by the planet, but that silicate atmospheres composed primarily of $\mathrm{Na}, \mathrm{O}_{2}, \mathrm{O}, \mathrm{Mg}$, and $\mathrm{SiO}$ gas may remain. The major atmospheric compounds are most likely to be $\mathrm{O}, \mathrm{Mg}$, and $\mathrm{SiO}$. The atmospheric composition may be altered by fractional vaporization, cloud condensation, photoionization, and a reaction with any residual volatile elements remaining in the atmosphere.

We also exclude the presence of a sodium torus like Io. The torus of Io around Jupiter may survive because there is the corotating plasma of Jupiter's plasma sphere. For CoRoT-7b, the ionized sodium would be quickly carried away by the stellar wind, so it is unlikely that a torus can form. It is thus unsurprising that we did not find a sodium torus.

In any case, for the first time we have been able to place constraints on the properties of an exosphere of a rocky extrasolar planet outside our solar system. This is particularly interesting, because CoRoT-7b does not resemble any solar-system rocky planet. Our observations also raise the question of how well it is justified to assume that this planet is a scaled-up version of objects in our solar system.

Acknowledgements. We are grateful to the User Support Group of ESO/Paranal. Part of this work was supported by a grant from the Deutschen Zentrums 
für Luft- und Raumfahrt (DLR) (50OW0204). H. Lammer, M. Fridlund, J. Schneider, A. Mura, and H. Rauer, also acknowledge the International Space Science Institute (ISSI; Bern, Switzerland) and the ISSI team "Evolution of Exoplanet Atmospheres and their Characterization". The authors also acknowledge support by the Europlanet FP7 project and fruitful discussions within the $\mathrm{Na} 2$ working groups WG4 and WG5.

\section{References}

Baliunas, S., Sokoloff, D., \& Soon, W. 1996, ApJ, 457, L99

Barnes, R., Raymond, S. N., Greenberg, R., Jackson, B., \& Kaib, N. A. 2010, ApJ, 709, L95

Bida, T. A., Killen, R. M., \& Morgan, T. H. 2000, Nature, 404, 159

Briot, D., \& Schneider, J. 2008, Poster at the conference Extra Solar Super-Earth, Nantes, June 16-18

Brown, R. A., Goody, R. M., Murcray, F. J., \& Chaffee, F. H., Jr. 1975, ApJ, 200 L49

Broadfoot, A. L., Shemansky, D. E., \& Kumar, S. 1976, Geophys. Res. Lett., 3, 577

Bruntt, H., Deleuil, M., Fridlund, M., et al. 2010, A\&A, 519, A51

Ekenbäck, A., Holmström, M., Wurz, P., et al. 2010, ApJ, 709, 670

Fossati, L., Haswell, C. A., Froning, C. S., et al. 2010, ApJ, 714, L222

Hatzes, A. P., Dvorak, R., Wuchterl, G., et al. 2010, A\&A, 520, A93

Holmström, M., Ekenbäck, A., Selsis, F., et al. 2008, Nature, 451, 970

Lecavelier Des Etangs, A., Ehrenreich, D., Vidal-Madjar, A., et al. 2010, A\&A 514, A72
Leitzinger, M., Odert, P., Lammer, H., et al. 2010, Planet. Space Sci. Sci., submitted

Linsky, J. L., Yang, H., France, K., et al. 2010, ApJ, 717, 1291

Léger, A., Rouan, D., Schneider, J., et al. 2009, A\&A, 506, 287

Mura, A., Wurz, P., Lichtenegger, H. I. M., et al. 2009, Icarus, 200, 1

Mohler et al. 2010, Exp. Astron., 28, 101

Mura, A., et al. 2010, Science, in press

Potter, A. E., \& Morgan, T. H. 1985, Science, 229, 651

Potter, A. E., Killen, R. M., \& Morgan, T. H. 2002, Meteoritics and Planetary Science, 37, 1165

Queloz, D., Bouchy, F., Moutou, C., et al. 2009, A\&A, 506, 303

Rauer, H., Bonev, T., Jockers, K., \& Thomas, N. 1993, Planet. Space Sci., 41, 1021

Schaefer, L., \& Fegley, B. 2009, ApJ, 703, L113

Schleicher, H., Wiedemann, G., Wöhl, H., Berkefeld, T., \& Soltau, D. 2004, A\&A, 425, 1119

Schneider, J., Boccaletti, A., Mawet, D., et al. 2009, Experimental Astron., 23, 357

Thomas, N. 1996, A\&A, 313, 306

Valencia, D., Ikoma, M., Guillot, T., \& Nettelmann, N. 2010, A\&A, 516, A20

Vidal-Madjar, A., Lecavelier des Etangs, A., Désert, J.-M., et al. 2003, Nature, 422,143

Vidal-Madjar, A., Désert, J.-M., Lecavelier des Etangs, A., et al. 2004, ApJ, 604, L69

Wurz, P. \& Lammer, H. 2003, Icarus, 164, 1

Wurz, P., Whitby, J. A., Rohner, U., et al. 2009, AGU Fall Meeting Abstracts, A1211 